\title{
G Protein-Coupled Receptors as Targets for Approved Drugs: How Many Targets and How Many Drugs? ${ }^{\mathrm{s}}$
}

\author{
Krishna Sriram and Paul A. Insel \\ Departments of Pharmacology (K.S., P.A.I.) and Medicine (P.A.I.), University of California San Diego, La Jolla, California
}

Received November 8, 2017; accepted December 21, 2017

\section{ABSTRACT}

Estimates vary regarding the number of $G$ protein-coupled receptors (GPCRs), the largest family of membrane receptors that are targeted by approved drugs, and the number of such drugs that target GPCRs. We review current knowledge regarding GPCRs as drug targets by integrating data from public databases (ChEMBL, Guide to PHARMACOLOGY, and DrugBank) and from the Broad Institute Drug Repurposing Hub. To account for discrepancies among these sources, we curated a list of GPCRs currently targeted by approved drugs. As of November 2017, 134 GPCRs are targets for drugs approved in the United States or European Union; 128 GPCRs are targets for drugs listed in the Food and Drug Administration Orange Book. We estimate that $\sim 700$ approved drugs target GPCRs, implying that approximately $35 \%$ of approved drugs target GPCRs. GPCRs and GPCR-related proteins, i.e., those upstream of or downstream from GPCRs, represent $\sim 17 \%$ of all protein targets for approved drugs, with GPCRs themselves accounting for $\sim 12 \%$. As such, GPCRs constitute the largest family of proteins targeted by approved drugs. Drugs that currently target GPCRs and GPCRrelated proteins are primarily small molecules and peptides. Since $\sim 100$ of the $\sim 360$ human endo-GPCRs (other than olfactory, taste, and visual GPCRs) are orphan receptors (lacking known physiologic agonists), the number of GPCR targets, the number of GPCR-targeted drugs, and perhaps the types of drugs will likely increase, thus further expanding this GPCR repertoire and the many roles of GPCR drugs in therapeutics.

\section{Introduction}

Based on their canonical structure, $G$ protein-coupled receptors (GPCRs), which are sometimes termed heptahelical or 7-transmembrane receptors, are the largest family of membrane receptors in humans and numerous other species. In addition, GPCRs are considered the largest family of targets for approved drugs (Allen and Roth, 2011; Rask-Andersen et al., 2014; Santos et al., 2017). Numerous factors contribute to the wide utility of GPCR-targeted drugs, including their druggability, interaction with numerous types of chemical entities, and expression in the plasma membrane, which facilitates molecular interactions in the extracellular milieu. Scientific articles, grant applications, and lectures that describe findings related to GPCRs often note the therapeutic utility of GPCRs but differ widely in estimates of their contribution as targets for approved drugs, which generally range from $\sim 20 \%$ to $>50 \%$. To

This work was supported by the American Society of Pharmacology and Experimental Therapeutics (ASPET) David Lehr Award, and by grants from the National Institutes of Health (R21AG52914, R21AG053568) and the Department of Defense (W81XWH-14-1-0372), and a contract with Bristol-Myers Squibb.

https://doi.org/10.1124/mol.117.111062.

S This article has supplemental material available at molpharm. aspetjournals.org. clarify this ambiguity, we reviewed information from three major public databases (see Materials and Methods). We report here the results of this analysis regarding the identity and number of GPCR targets and drugs that target those GPCRs, as well as GPCR-related proteins upstream (e.g., ligands) and downstream (e.g., phosphodiesterases [PDEs] that degrade cAMP). In addition, we provide information about the types of chemical entities that are GPCR-directed therapeutics and identify inconsistencies among the databases regarding GPCR targets and drugs directed at those targets.

\section{Materials and Methods}

We assessed information regarding approved drugs, drug targets, and if available ligand-target interactions from three online databases: ChEMBL (Bento et al., 2014), DrugBank (Wishart et al., 2007), and the International Union of Basic and Clinical Pharmacology/BPS (British Pharmacological Society) Guide to PHARMACOLOGY (GtoPdb) (Southan et al., 2016; Alexander et al., 2017). We consolidated data from each source into tables that relate the drugs approved for use in humans to their targets. We obtained a list of all annotated human GPCRs (excluding olfactory and vision receptors) from GtoPdb (Alexander et al., 2017); the genes/proteins on this list were then queried from different sources to identify GPCRs that are drug targets. Approved drugs were cross-checked against those listed by 
the Food and Drug Administration (FDA) (fda.gov) and European Medicines Agency (EMA) (ema.europa.eu/ema/). As an additional source, we used data from the Broad Institute Drug Repurposing Hub (Corsello et al., 2017), which employed high-throughput screening to characterize drug-target interactions of approved drugs, natural products, and nutraceuticals along with other entities.

Our analysis yielded a list of currently druggable GPCRs and the drugs that target them. Because of possible database errors in downloadable material, as well as ambiguity of ligand-target interactions in certain sources, we manually curated each druggable GPCR to verify pharmacological interaction of the approved drugs. The primary sources used for crosschecking the data were the aforementioned databases, plus the Drug Gene Interaction Database (dgidb.org; Cotto et al, 2017) and GeneCards (genecards.org; Rebhan et al., 1998), along with primary sources cited within these databases.

The resulting list of GPCRs targeted by approved drugs (Table 1) includes an example of an approved drug that targets each GPCR. Certain GPCRs (for example, histamine H1) are the targets for multiple drugs. Supplemental Table 2 lists all such GPCR-targeted drugs. In some cases, we considered families of GPCRs as targets for the same drugs (e.g., multiple $\mathrm{P} 2 \mathrm{Y}$ receptors are targets for suramin and multiple $\mathrm{S} 1 \mathrm{P}$ receptors as targets for fingolimod). For such examples, we list the GPCRs and approved drugs with the caveat that some of the drugGPCR interactions are not well defined. In certain cases (e.g., suramin), the approval status of a drug is unclear; we provide details for such drugs in Table 1 and the accompanying legend.

We thus assembled an integrated list of approved drugs that target GPCRs by combining data from the previously noted databases (ChEMBL, GtoPdb, and DrugBank) and the Broad Drug Repurposing Hub. Due to difficulties in cross-referencing information among the databases (since common identifiers such as Chemical Abstracts Service numbers are not provided for drugs listed by all sources), we generated lists of GPCR-related drugs using drug names. We considered drugs with multiple derivatives (such as different salts) as duplicates in counting GPCR-related drugs and evaluating overlap/discrepancy among sources. In some cases, the aforementioned sources list drugs approved in the United States and/or Europe but without noting the approving agency. We thus estimated the number of GPCR-targeting drugs based on their approval status, without including information regarding the agencies that have approved such drugs, although the majority of such drugs listed in Supplemental Table 2 are listed in the FDA Orange Book.

\section{Results}

GPCRs That Are Targets for Approved Drugs. Table 1 lists GPCRs that are targets for approved drugs, and for each GPCR we identify an example of an approved drug. We identified 134 GPCRs that are targets for approved drugs; this number represents about one-third of the endo-GPCR family, i.e., $n=364$ (Supplemental Table 1) (Insel et al., 2015; Alexander et al., 2017), but only $\sim 16 \%$ of the $~ 800$ GPCRs (including olfactory receptors) in the human genome. The currently druggable receptors include GPCRs that signal via each class of heterotrimeric GTP $(\mathrm{G})$ proteins (Gs, Gi/o, Gq/11, and G12/13), and are members of each of the major families of GPCRs: classes A, B, C, frizzled, and adhesion (Fig. 1). SMO, of the frizzled class and ADGRG3, an adhesion receptor, are the only GPCRs among these 134 druggable receptors without known physiologic agonists. A small number of GPCRs interact with approved drugs but lack well-characterized $G$ protein-mediated signaling, for example, GPR35 is targeted by furosemide and bumetanide (Yang et al., 2012), both of which bind this GPCR with similar affinity to SLC12 chloride transporters - considered the primary targets of these drugs (Hebert et al., 2004).
Numerous approved drugs are physiological agonists for GPCRs (for example, epinephrine [at multiple adrenergic receptors] and numerous peptide hormones). Entries in bold in Table 1 indicate GPCRs considered primary targets for the listed drug, based on affinity and/or functional response (in the majority of cases, both). Entries not in bold indicate GPCRs targeted by approved drugs but for which such an interaction is not likely a primary aspect of the intended therapeutic use of such drugs. Such GPCR-ligand interactions may contribute to side effects. In a number of cases, data are ambiguous regarding precisely which receptors are responsible for mediating the therapeutic efficacy of GPCR-targeted drugs. In such cases, if pharmacological data implicate a strong GPCR-drug affinity, we list GPCR-drug pairs in bold. An example is the melanocortin receptor family (MC1R-MC5R), which may mediate a number of therapeutic effects of corticotropin/ adrenocorticotropin (ACTH; Gong, 2014). Similarly, the precise importance of each of the somatostatin receptors as targets for pasireotide and lanreotide is incompletely understood (e.g., Feelders et al., 2012). In total, we thus list 98 GPCRs that are thought to mediate therapeutic effects of approved drugs, with an additional 36 GPCRs that interact with approved drugs and may contribute to therapeutic effects or side effects. We excluded multiple GPCRs as primary targets for molecules with affinity for multiple members within one or more GPCR families. As examples, caffeine and theophylline bind multiple adenosine receptors including ADORA2B, but we do not consider that receptor to be a primary target for any approved drugs. Our rationale is similar for suramin, which binds multiple P2Y receptors.

Different databases/sources identify differing numbers and identities of GPCRs that are targets of approved drugs. Figure 1A shows the number of druggable GPCRs identified by each source we used. 157 GPCRs are identified by one or more source as a target for approved drugs. Three of the four sources indicate a similar number of GPCRs as druggable but the identities of such receptors are discrepant. ChEMBL identifies fewer GPCRs than the other sources, as we will discuss subsequently. A small number of GPCRs are uniquely identified in each of the four sources (see the Fig. 1 legend). Supplemental Table 1 lists the druggable GPCRs identified by each source.

Figure 1B illustrates the primary $\mathrm{G} \alpha$ protein linkage for the 134 GPCRs with approved drugs. In cases where GPCRs couple to multiple $\mathrm{G} \alpha$ linkages, we counted all such linkages. Approximately two-thirds of the targeted receptors couple to either Gs or Gi, thereby highlighting the importance of such GPCRs (and likely, the regulation by cAMP) of current therapeutic agents. By contrast, approved drugs only target eight GPCRs that signal via G12/13. Two examples are F2R/PAR1, the thrombin receptor, targeted by vorapaxar, and SMO, targeted by sonidegib and vismodegib. Such assignments may change as data emerge that identify drugs with signaling bias or activity as allosteric modulators. For example, the benzodiazepine lorazepam, whose anxiolytic action results from interaction with $\mathrm{GABA}_{\mathrm{A}}$ receptors, also binds to the $\mathrm{pH}$-sensing GPCR GPR68/OGR1 and promotes Gq-mediated signaling (Huang et al., 2015a; Pera et al., 2017).

The GtoPdb, DrugBank, and Broad Institute Drug Repurposing Hub data sets show relatively good agreement. For example, as shown in Fig. 1C, GtoPdb and DrugBank share in their identification of $>80 \%$ of GPCRs. The Broad data set has the largest number of GPCR drug targets, likely resulting 
TABLE 1

One hundred thirty-four GPCRs have FDA-approved drugs

Exceptions are those marked as follows: *, EMA approved; 1, approved in the United Kingdom by the The Medicines and Healthcare products Regulatory Agency (MHRA); 2 , suramin is not listed in the FDA's Orange Book, but is distributed by the Centers for Disease Control and is listed as an essential medicine by the World Health Organization; and \#, GABBR1 and 2 combine to form a single active signaling complex that is activated by baclofen. Entries in bold in indicate GPCRs considered primary targets for the listed drug based on affinity and/or functional response (in the majority of cases, both). Entries not in bold indicate GPCRs targeted by approved drugs but for which such an interaction is not likely a primary aspect of the intended therapeutic use of such drugs.

\begin{tabular}{|c|c|}
\hline Gene Name & Example of an Approved Drug \\
\hline ACKR3 & Plerixafor \\
\hline$A D G R G 3$ & Beclometasone dipropionate \\
\hline ADORA1 & Adenosine \\
\hline ADORA2A & Regadenoson \\
\hline$A D O R A 2 B$ & Theophylline \\
\hline$A D O R A 3$ & Nicardipine \\
\hline$A D R A 1 A$ & Oxymetazoline \\
\hline$A D R A 1 B$ & Prazosin \\
\hline$A D R A 1 D$ & Prazosin \\
\hline ADRA2A & Apraclonidine \\
\hline$A D R A 2 B$ & Dexmedetomidine \\
\hline$A D R A 2 C$ & Dexmedetomidine \\
\hline$A D R B 1$ & Acebutolol \\
\hline$A D R B 2$ & Pindolol \\
\hline$A D R B 3$ & Mirabegron \\
\hline AGTR1 & Candesartan \\
\hline AVPR1A & Vasopressin \\
\hline AVPR1B & Vasopressin \\
\hline AVPR2 & Vasopressin \\
\hline$B D K R B 1$ & Icatibant \\
\hline BDKRB2 & Icatibant \\
\hline CALCR & Calcitonin \\
\hline CASR & Etelcalcetide \\
\hline CCKAR & Ceruletide \\
\hline$C C K B R$ & Pentagastrin \\
\hline CCR4 & Plerixafor \\
\hline CCR5 & Maraviroc \\
\hline CHRM1 & Biperiden \\
\hline CHRM2 & Propantheline \\
\hline CHRM3 & Umeclidinium \\
\hline CHRM4 & Acetylcholine \\
\hline CHRM5 & Acetylcholine \\
\hline CNR1 & Nabilone \\
\hline CNR2 & Nabilone \\
\hline CRHR1 & Corticorelin ovine triflutate \\
\hline CXCR4 & Plerixafor \\
\hline CYSLTR1 & Zafirlukast \\
\hline CYSLTR2 & Zafirlukast \\
\hline $\mathrm{DRD1}$ & Dopamine \\
\hline DRD2 & Dopamine \\
\hline DRD3 & Dopamine \\
\hline $\mathrm{DRD4}$ & Dopamine \\
\hline DRD5 & Dopamine \\
\hline EDNRA & Ambrisentan \\
\hline$E D N R B$ & Bosentan \\
\hline F2R & Vorapaxar \\
\hline FFAR1 & Rosiglitazone \\
\hline FPR1 & Cyclosporine \\
\hline FSHR & Human follicle stimulating hormone \\
\hline GABBR1 \# & Baclofen \\
\hline$G A B B R 2$ \# & Baclofen \\
\hline GCGR & Glucagon \\
\hline GHRHR & Sermorelin \\
\hline GLP1R & Lixisenatide \\
\hline GLP2R & Teduglutide \\
\hline GNRHR & Abarelix \\
\hline GPBAR1 & Deoxycholic acid \\
\hline GPER1 & Estradiol \\
\hline$G P R 143$ & Levodopa \\
\hline$G P R 18$ & Dronabinol \\
\hline GPR35 & Bumetanide \\
\hline GPR55 & Dronabinol \\
\hline GPR68 & Lorazepam \\
\hline$H C A R 1$ & Sodium oxybate \\
\hline
\end{tabular}

(continued)
TABLE 1-Continued

\begin{tabular}{|c|c|}
\hline Gene Name & Example of an Approved Drug \\
\hline HCAR2 & Acipimox 1 \\
\hline$H C A R 3$ & Nicotinic acid \\
\hline HCRTR1 & Suvorexant \\
\hline HCRTR2 & Suvorexant \\
\hline HRH1 & Cetirizine \\
\hline HRH2 & Betazole \\
\hline HRH3 & Pitolisant $*$ \\
\hline HRH4 & Clozapine \\
\hline HTR1A & Vilazodone \\
\hline HTR1B & Frovatriptan \\
\hline HTR1D & Frovatriptan \\
\hline HTR1E & Asenapine \\
\hline HTR1F & Eletriptan \\
\hline HTR2A & Asenapine \\
\hline HTR2B & Methysergide \\
\hline HTR2C & Methysergide \\
\hline HTR4 & Cisapride \\
\hline HTR5A & Ergotamine \\
\hline HTR6 & Amoxapine \\
\hline HTR7 & Lurasidone \\
\hline LHCGR & Choriogonadotropin alfa \\
\hline MC1R & Corticotropin \\
\hline$M C 2 R$ & Corticotropin \\
\hline$M C 3 R$ & Corticotropin \\
\hline$M C 4 R$ & Corticotropin \\
\hline MC5R & Corticotropin \\
\hline$M L N R$ & Erythromycin \\
\hline$M R G P R X 1$ & Chloroquine \\
\hline MTNR1A & Ramelteon \\
\hline MTNR1B & Tasimelteon \\
\hline NPY4R & Niclosamide \\
\hline NTSR2 & Levocabastine \\
\hline OPRD1 & Naltrexone \\
\hline OPRK1 & Anileridine \\
\hline OPRM1 & Alfentanil \\
\hline OXTR & Oxytocin \\
\hline$P 2 R Y 1$ & Suramin 2 \\
\hline$P 2 R Y 11$ & Suramin 2 \\
\hline P2RY12 & Cangrelor \\
\hline$P 2 R Y 13$ & Cangrelor \\
\hline P2RY2 & Suramin 2 \\
\hline P2RY6 & Suramin 2 \\
\hline$P T G D R$ & Treprostinil \\
\hline PTGDR2 & Indomethacin \\
\hline PTGER1 & Prostaglandin E1 \\
\hline PTGER2 & Prostaglandin E2 \\
\hline PTGER3 & Misoprostol \\
\hline PTGER4 & Treprostinil \\
\hline PTGFR & Latanoprost \\
\hline PTGIR & Epoprostenol \\
\hline PTH1R & Teriparatide \\
\hline PTH2R & Teriparatide \\
\hline S1PR1 & Fingolimod \\
\hline S1PR2 & Fingolimod \\
\hline S1PR3 & Fingolimod \\
\hline S1PR4 & Fingolimod \\
\hline S1PR5 & Fingolimod \\
\hline SCTR & Secretin \\
\hline SMO & Sonidegib \\
\hline SSTR1 & Pasireotide \\
\hline SSTR2 & Lanreotide \\
\hline SSTR3 & Pasireotide \\
\hline SSTR4 & Octreotide \\
\hline SSTR5 & Lanreotide \\
\hline SUCNR1 & Sodium succinate \\
\hline$T A A R 1$ & Dexamfetamine \\
\hline$T A C R 1$ & Aprepitant \\
\hline$T B X A 2 R$ & Iloprost \\
\hline TRHR & Protirelin \\
\hline TSHR & Thyrotropin \\
\hline
\end{tabular}

from the use of different inclusion criteria compared with the other sources, such as by including a large number of natural products not listed in other sources. However, certain drugtarget interactions in the Broad data set likely do not result 

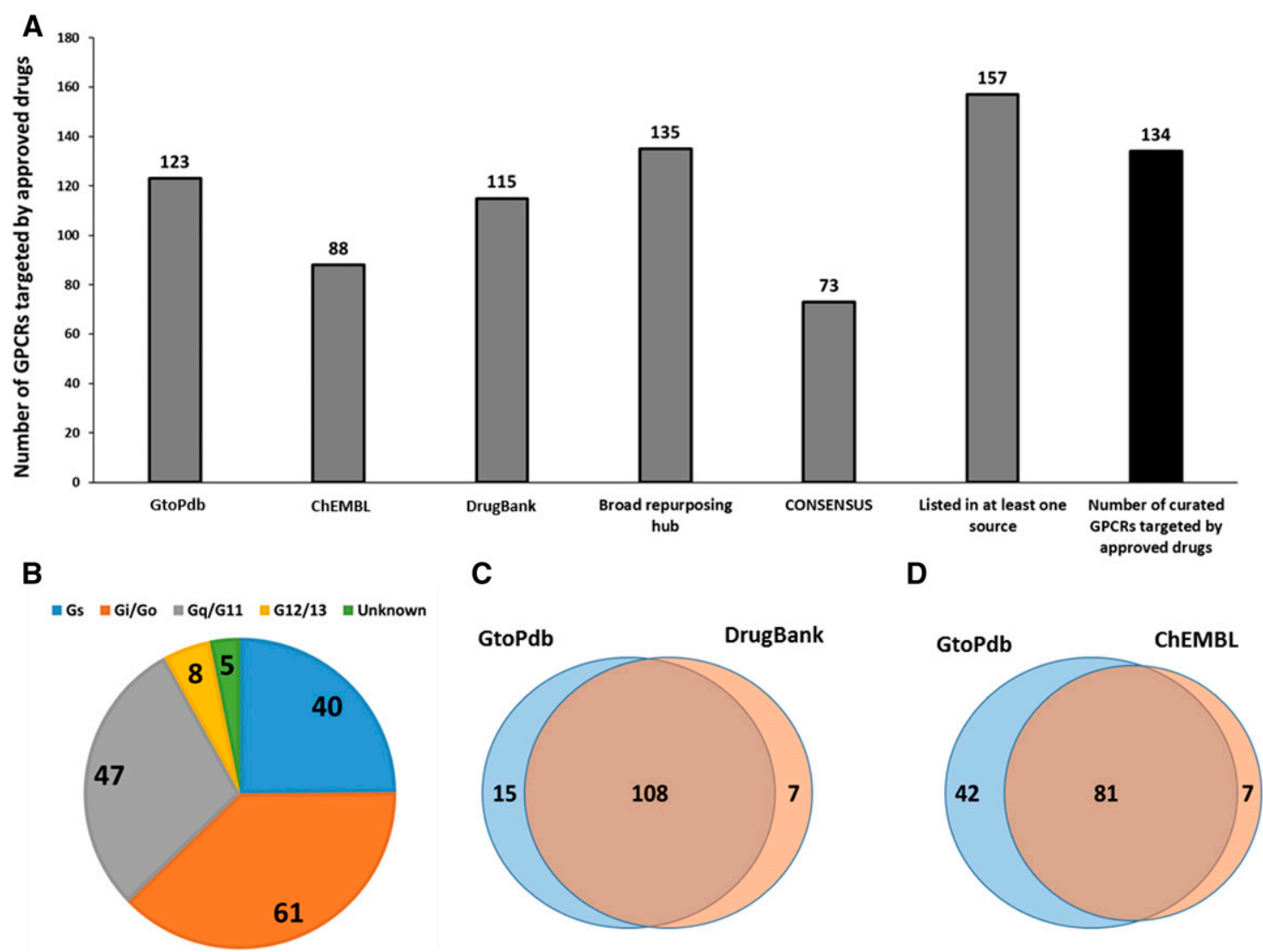

Fig. 1. The number of GPCRs targeted by approved drugs. (A) The number of GPCRs targeted by approved drugs, based on annotations of GPCR-drug interactions from each of several sources. Also included is the number of commonly listed (consensus) GPCRs and GPCRs curated in our analysis. The number of druggable GPCRs uniquely listed in each source is as follows: GtoPdb, 6; ChEMBL, 3; DrugBank, 0; and Broad Institute Drug Repurposing Hub, 24. (B) Putative primary G $\alpha$ protein linkage, based on classification of GPCR signaling by GtoPdb, of the 134 curated GPCRs targeted by approved drugs. Individual GPCRs may couple to multiple G-proteins. (C and D) Venn diagrams of the GtoPdb's list of GPCR drug targets and that of ChEMBL and DrugBank.

from ligand-receptor interaction. For example, that data set lists GPRC5A as a target of retinoic acid. However, retinoic acid does not bind GPRC5A; instead, retinoic acid regulates its expression (Ye et al., 2009). In the absence of data indicating drug-receptor interaction, we believe a GPCR should not be considered as a target for an approved drug.

Other discrepancies, in particular between GtoPdb and ChEMBL (Fig. 1D), appear to result from drugs that have differing affinities in their interaction with multiple targets. The GtoPdb database tends to list all GPCRs with at least moderate affinity for such drugs, whereas the ChEMBL database lists only the primary target. An example is nabilone, an FDA-approved synthetic cannabinoid, which targets $\mathrm{CB} 1$ and CB2 receptors but with only slightly higher ( 2-fold) affinity for CB1. GtoPdb lists both $\mathrm{CB} 1$ and $\mathrm{CB} 2$ receptors as drug targets, whereas ChEMBL lists only CB1. Other such discrepancies include $\mathrm{B} 1$ and $\mathrm{B} 2$ bradykinin receptors, which are targeted by icatibant, for which the $\mathrm{B} 2$ receptor has a higher affinity: only B2 is listed as druggable in ChEMBL but both are considered drug targets in GtoPdb. In such cases, we have used GtoPdb's approach, i.e., listing all GPCRs with a reasonable affinity for approved drugs as druggable, while also distinguishing between primary and secondary targets as discussed previously. We curated data for each GPCR to ensure availability of pharmacological data for at least one drug approved either in the United States (FDA) or Europe (EMA) to define currently druggable GPCRs. Of the 134 such GPCRs we curated (Table 1), 128 are targets of FDA-approved drugs. Of the remaining GPCRs, for the four P2Y receptors (P2YR 1, 2, 6, and 11), suramin is an antagonist but is not formally approved by the FDA, yet it is listed as an essential medicine by the World Health Organization and distributed by the Centers for Disease Control as an anti-parasitic agent. Two other such GPCRs are HCAR2, a target for acipimox (UKapproved), and HRH3, a target for the antagonist pitolisant (EMA approved).

FDA-Approved Drug Targets Upstream of or Downstream from GPCRs. In addition to drugs that bind/interact with GPCRs, numerous approved drugs target proteins, such as transporters and enzymes that are proximal (upstream) or distal (downstream) of GPCRs and thereby contribute to GPCR activation and function. Examples include agents that regulate agonist availability by controlling ligand synthesis or transport (e.g., serotonin or norepinephrine reuptake inhibitors) or proteins that regulate concentration of second messengers, for example, inhibitors of PDEs (such as the PDE4 inhibitors roflumilast and apremilast), that hydrolyze cAMP. Calcium is a second messenger for Gq signaling but we have not included proteins that regulate cellular calcium concentrations (such as voltage-gated ion channels) since these 
TABLE 2

GPCR-related proteins/genes that are targets for approved drugs

\begin{tabular}{|c|c|}
\hline Examples of Proteins/Genes That Are Drug Targets & $\begin{array}{l}\text { Examples of } \\
\text { Drugs That } \\
\text { Target These } \\
\text { Proteins/Genes }\end{array}$ \\
\hline \multicolumn{2}{|l|}{ Protein/gene category: enzymes involved in agonist synthesis } \\
\hline Prostaglandin I2 synthase & Epoprostenol \\
\hline Angiotensin I converting enzymes & Lisinopril \\
\hline Dopamine beta-hydroxylase & Hydralazine \\
\hline Coagulation factors (e.g., factor 10) & Rivaroxaban \\
\hline \multicolumn{2}{|c|}{ Protein/gene category: enzymes involved in agonist degradation } \\
\hline Monoamine oxidases $\mathrm{A}$ and $\mathrm{B}$ & Phenelzine \\
\hline Catechol $O$-methyltransferase & Entacapone \\
\hline Acetylcholinesterase & Neostigmine \\
\hline Adenosine deaminase & Pentostatin \\
\hline Dipeptidyl peptidase-4 & Sitagliptin \\
\hline \multicolumn{2}{|l|}{ Protein/gene category: transporters of GPCR agonists } \\
\hline Serotonin transporters & Citalopram \\
\hline Dopamine transporters (SLC6A3) & Modafinil \\
\hline ATP transporters (SLC25A4) & Clodronic acid \\
\hline \multicolumn{2}{|l|}{ Protein/gene category: GPCR ligands } \\
\hline C-C motif chemokine ligand 2 & Mimosine \\
\hline Coagulation factor II, thrombin & Argatroban \\
\hline \multicolumn{2}{|c|}{ Protein/gene category: regulators of second messenger signaling } \\
\hline PDEs & \\
\hline PDE1A-C & Vinpocetine \\
\hline PDE4A-D & Roflumilast \\
\hline Rho Kinases (ROCK1-2) & Fasudil \\
\hline
\end{tabular}

proteins generally regulate calcium in contexts independent of GPCR signaling. We also have excluded genes/proteins associated with metabolism (such as in the Krebs cycle) since the primary functions of such proteins are only indirectly related to GPCR signaling. Thus, Table 2 lists GPCR-related drug targets that are proteins whose function/activity is primarily/largely dependent on GPCRs, and whose targeting directly influences GPCR signaling.

We identified over 50 druggable GPCR-related proteins. Adding these proteins to the list of GPCRs yields $>180$ targets for approved drugs. Estimates of the total number of druggable genes/proteins vary. For example, Santos et al. (2017) recently estimated nearly 700 druggable genes, whereas the three major databases (ChEMBL, GtoPdb, and DrugBank) each list $\sim 800$ druggable genes. A combined list from those databases yields $\sim 1100$ human gene/protein targets for approved drugs. Such estimates lead us to propose that GPCRs and GPCR-related genes together account for $\sim 17 \%$ of the genes/proteins currently targeted by approved drugs (Fig. 2). GPCRs alone represent $\sim 12 \%$ of drug targets and hence are the largest family of genes/proteins targeted by approved drugs, followed by voltage-gated ion channels and protein kinases. To verify which of the druggable genes are in different categories, against the 1100 druggable genes, we queried lists of all genes annotated as protein kinases (from UniProt) (The UniProt Consortium, 2017), voltage-gated ion channels, ligand-gated ion channels, and transporters (all from GtoPdb) (Southan et al., 2016).

Approved Drugs That Target GPCRs. Substantial discrepancy exists among the four main sources used in this study with respect to the numbers and identities of approved drugs that target GPCRs (Fig. 3A). A major challenge in comparing these databases is the lack of suitable fields for cross-referencing among sources. DrugBank, GtoPdb, and ChEMBL do not use the same identifiers for drugs (such as Chemical Abstracts Service numbers) in downloadable data files. ChEMBL provides a cross-referencing method via the UniChem web API tool (Chambers et al., 2013); however, this still did not allow for thorough cross-referencing since several compounds are listed in GtoPdb or DrugBank but not ChEMBL/UniChem. Thus, it is problematic to readily convert between GtoPdb ligand, ChEMBL, and Drugbank identifiers, a difficulty also noted by others, e.g., Santos et al. (2017), who also used drug names rather than a universal identifier when compiling data from sources external to their own databases. Compiling drug names among the different sources yields 704 drugs annotated by at least one source as targeting one or more of the 134 druggable GPCRs listed in Table 1. Supplemental Table 2 lists all such drugs, as well as the source that lists them. Of these 704 drugs, 199 are annotated as targeting GPCRs in only a single source, most commonly, in the Broad

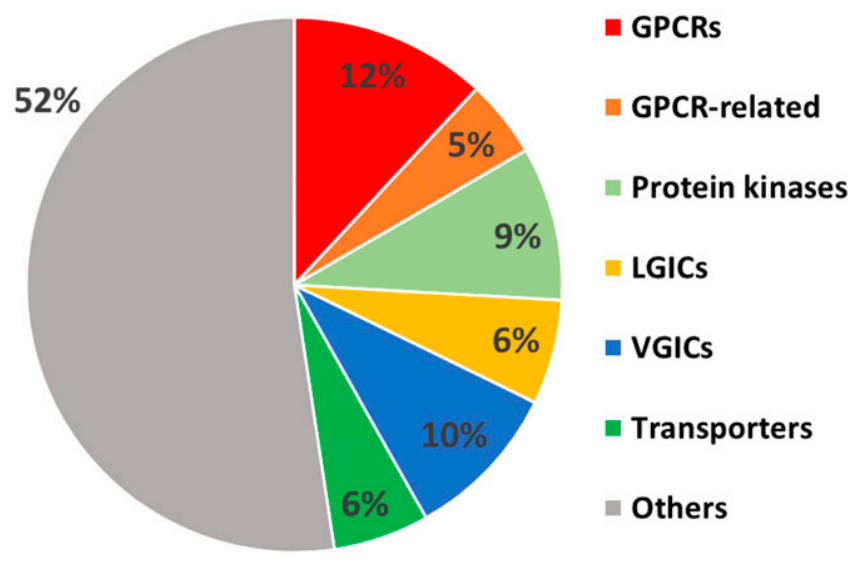

Fig. 2. The estimated proportion of genes from different gene families that are targets for approved drugs. GPCRs comprise the single largest such group. VGICs: voltage-gated ion channels; LGICs: ligand-gated ion channels. 
A

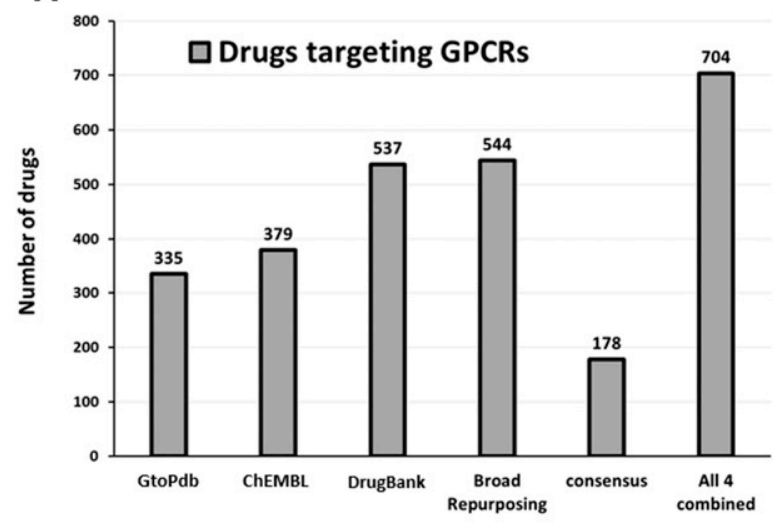

C

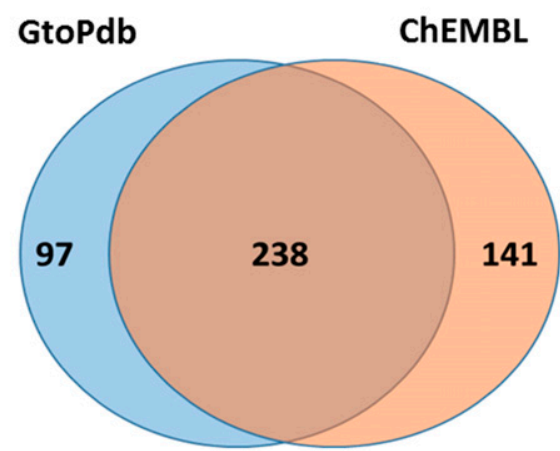

B

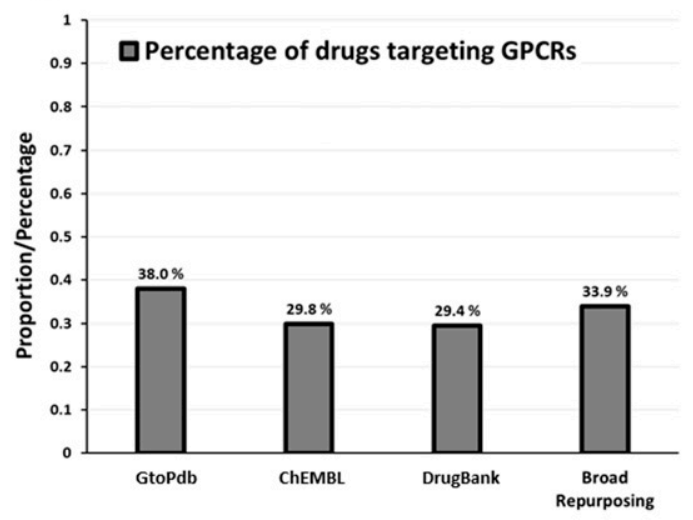

D

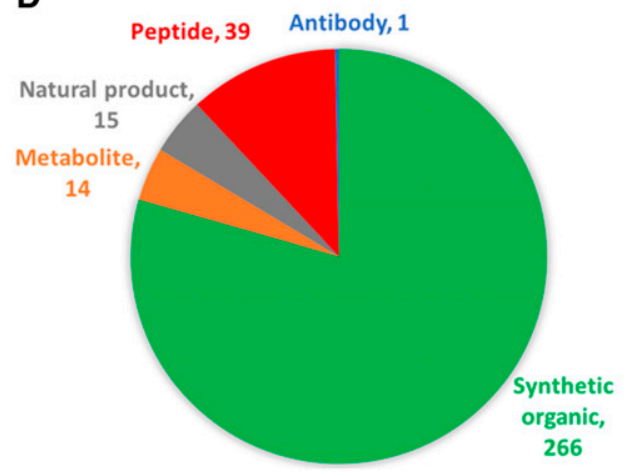

Fig. 3. The number of GPCR-targeted drugs. (A) The number of approved drugs identified in GtoPdb, CHEMB, DrugBank, and the Broad repurposing hub with the consensus among the four sources and each entry combined from the four sources. (B) The percentage of approved drugs that target GPCRs in each database. (C) Venn diagram showing the relationship between identities of GPCR-related drugs listed in the GtoPdb and ChEMBL databases. (D) The types of molecules that target GPCRs, based on annotations from GtoPdb (Alexander et al., 2017).

Drug Repurposing Hub data or DrugBank. Among these 704 drugs, we included entries from DrugBank for which DrugBank provide information on pharmacological interactions or where such data are available in other data sets.

Estimates of the proportion of approved drugs that target GPCRs vary; the ChEMBL and DrugBank databases each indicate $\sim 30 \%$ of drugs target GPCRs, while the GtoPdb database lists $\sim 38 \%$ (Fig. 3B). Together, the FDA and EMA list $\sim 1950$ approved drugs. Using a range of $\sim 500$ (conservative estimate) to $\sim 700$ GPCR-targeted drugs, we estimate that between $\sim 25 \%$ and $\sim 36 \%$ of approved drugs target GPCRs, with the upper figure the more likely. As additional studies such as the Broad repurposing initiative (Corsello et al., 2017) characterize GPCR-drug interactions in more detail, we anticipate a growth in this number, as secondary interactions between GPCRs and drugs are defined (Allen and Roth, 2011).

GtoPdb lists more druggable GPCRs than ChEMBL or DrugBank but has the smallest number of GPCR-related and overall approved drugs (Fig. 3, A and B). Figure 3C shows the number of GPCR-targeted drugs based on target-ligand interactions annotated by either GtoPdb or ChEMBL: of the 476 such drugs listed in one or both sources, only a portion are common to both (50\%). Such discrepancies highlight the need for more detailed and accurate annotation of druggable genes/proteins and the drugs that target them, as well as measures that will facilitate cross-referencing among databases to reconcile such inconsistencies.
Another issue is that different sources categorize/classify types of drugs differently. GtoPdb provides the most detailed breakdown, classifying drugs as either peptides, antibodies, natural products, metabolites, or synthetic organics (typically, small molecules). Figure 3D shows the classes of GPCRtargeted drugs listed by GtoPdb. Most are synthetic organic molecules. Antibodies represent a novel class of therapeutics for GPCRs. Mogamulizumab, a CCR4 antagonist, is currently the only approved antibody that targets a GPCR, although $>70$ clinical programs on GPCR antibodies are in progress (Hutchings et al., 2017).

Which GPCRs Are Targeted by the Most Approved Drugs? Reflective of the discrepancies between databases, the number of drugs that most frequently target GPCRs varies widely among the sources (Fig. 4). Histamine (HRH1), serotonin, dopamine, opioid, and adrenergic receptors are the most frequently targeted GPCRs, in terms of the number of available drugs. DrugBank lists a larger number of drugs interacting with many GPCRs, likely because DrugBank has the largest number of annotated drugs and drug-target interactions and also has less strict inclusion criteria with respect to ligand-target binding data in considering a protein target for a given drug. The large number of HRH1 antagonists reflects their frequent use in treating allergic responses (Xie and $\mathrm{He}$, 2005). That multiple members of certain families of GPCRs are druggable raises the possibility that other families of GPCRs, including orphan GPCRs, may also become druggable 

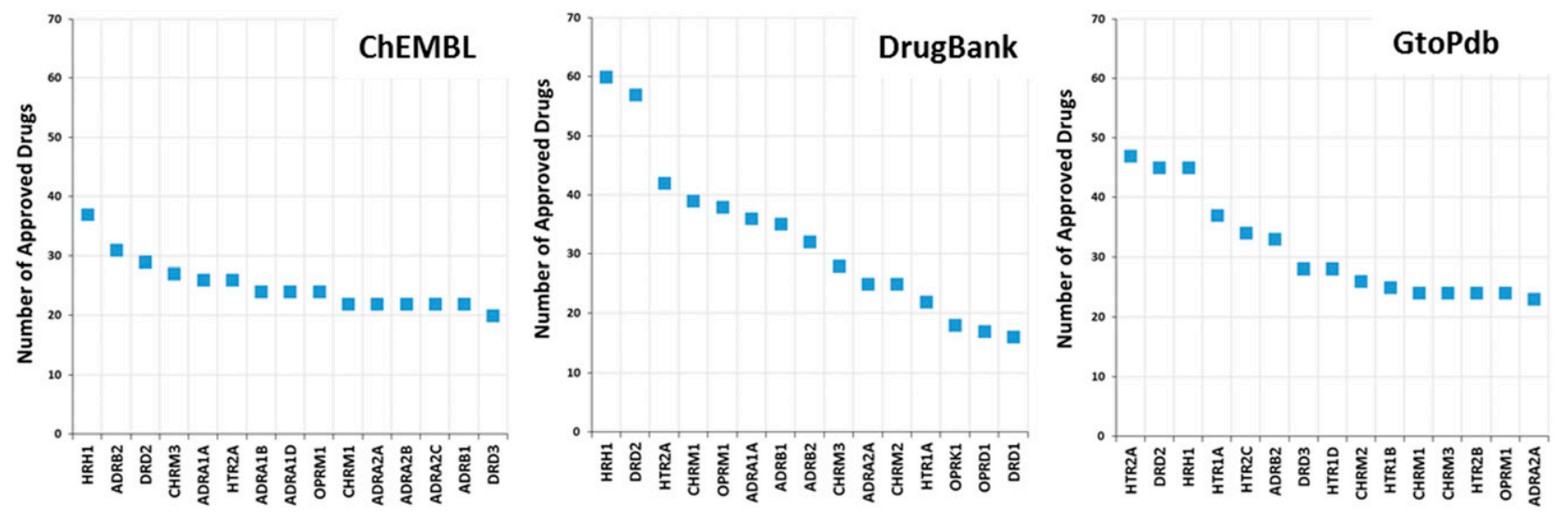

Fig. 4. The 15 GPCRs with the most approved drugs and the number of such drugs listed in ChEMBL, DrugBank, and GtoPdb.

(Huang et al., 2015a). Supplemental Table 3 lists approved drugs that target each GPCR from each major source surveyed.

\section{Discussion}

Our review of information in databases confirms that: 1) GPCRs represent the largest family of protein targets for approved drugs and 2) GPCRs are the family of protein targets most frequently targeted by approved drugs (see section on FDA-Approved Drug Targets Upstream of or Downstream from GPCRs). Previous studies (e.g., Santos et al., 2017) have estimated the contribution of GPCRs to the druggable genome. By curating information from public databases, we have identified 134 GPCRs that are targets for currently approved drugs, in particular, FDA-approved drugs. Approximately two-thirds of currently druggable GPCRs regulate cAMP and numerous GPCR-related drug targets influence cAMP signaling (e.g., PDEs or proteins associated with synthesis/ transport of ligands whose receptors regulate cAMP synthesis). The cAMP pathway is thus likely the most frequently targeted signaling pathway by currently approved therapeutics. It should, however, be noted that these cAMP-regulating GPCRs and other proteins may also signal via cAMPindependent mechanisms; e.g., Gi-coupled GPCRs may signal via $\beta \gamma \mathrm{G}$ protein subunits (which regulate a variety of pathways) or via $\beta$-arrestin. Biased signaling may also influence estimates for how many GPCRs from each signaling category are targets for drugs.

Discrepancies among databases are a concern for both the number of GPCR targets in different sources and of drugs that target GPCRs. We suggest that these problems can be addressed by collaborative, expert review of the content in databases, akin to the approach used by GtoPdb to involve experts to annotate and curate data for drug targets (Alexander et al., 2017). Recent efforts by groups such as the Drug Gene Interaction Database (Cotto et al, 2017) have sought to consolidate data from different sources into single, searchable data sets. A combination of individually curated and broadly consolidated data may help solve the challenge of developing comprehensive databases with agreed upon data for drugs and drug targets.

With the exception of SMO and ADGRG3, orphan GPCRs (without known physiologic agonists) are not currently drug targets. Certain orphan GPCRs have highly selective patterns of expression or activity in normal and disease tissue. Examples include GPRC5A in pancreatic cancer (Jahny et al., 2017), GPR22 in heart disease (Patel and Ostrom, 2008), SMO as part of hedgehog signaling in various cancers (Rimkus et al., 2016), and GPR3 in Alzheimer's disease (Huang et al., 2015b). Such receptors represent a potentially fruitful area for further study and may add to the repertoire of druggable GPCRs and the drugs that target them. In addition, efforts to design new types of GPCR-targeted drugs (e.g., with signaling bias [via $\mathrm{G}$ proteins or $\beta$-arrestin] and as allosteric modulators), and with new types of therapeutic agents (including antibodies/nanobodies, aptamers, anti-sense oligonucleotides, and gene therapies), and with ligands delivered in novel ways, lead us to predict that GPCRs will continue to play a prominent role as therapeutic targets. Evidence that GPCRs can show differential expression in diseased cells and tissues will likely underlie efforts to design and develop new GPCRtargeted drugs.

Data such as those presented here may allow for more accurate evaluation of interactions and off-target effects of approved drugs with GPCRs (Allen and Roth, 2011; Wacker et al., 2017), as well as providing opportunities for novel repurposing of approved drugs. As noted previously, an example is the anti-anxiety drug lorazepam, which is an allosteric modulator of GPR68, a proton-sensing GPCR (Huang et al., 2015a). Work from our laboratory has identified a role for this receptor in pancreatic cancer (Wiley et al., 2017); raising the possibility that lorazepam (or perhaps other benzodiazepines) may be repurposed for this disease. Data such as those presented in this review may thus not only help identify new potential targets for basic science studies, but may also reveal novel clinically relevant therapeutic opportunities.

\section{Authorship Contributions}

Participated in research design: Sriram, Insel.

Performed data analysis: Sriram.

Wrote or contributed to the writing of the manuscript: Sriram, Insel.

\section{References}

Alexander SPH, Kelly E, Marrion NV, Peters JA, Faccenda E, Harding SD, Pawson AJ, Sharman JL, Southan C, Buneman OP, et al.; CGTP Collaborators (2017) The concise guide to pharmacology 2017/18: overview. Br J Pharmacol 174 (Suppl 1): S1-S16. 
Allen JA and Roth BL (2011) Strategies to discover unexpected targets for drugs active at G protein-coupled receptors. Annu Rev Pharmacol Toxicol 51:117-144.

Bento AP, Gaulton A, Hersey A, Bellis LJ, Chambers J, Davies M, Krüger FA, Light Y, Mak L, McGlinchey S, et al. (2014) The ChEMBL bioactivity database: an update. Nucleic Acids Res 42:D1083-D1090.

Chambers J, Davies M, Gaulton A, Hersey A, Velankar S, Petryszak R, Hastings J, Bellis L, McGlinchey S, and Overington JP (2013) UniChem: a unified chemical structure cross-referencing and identifier tracking system. J Cheminform 5:3.

Corsello SM, Bittker JA, Liu Z, Gould J, McCarren P, Hirschman JE, Johnston SE Vrcic A, Wong B, Khan M, et al. (2017) The Drug Repurposing Hub: a nextgeneration drug library and information resource. Nat Med 23:405-408.

Cotto KC, Wagner AH, Feng YY, Kiwala S, Coffman AC, Spies G, Wollam A, Spies NC, Griffith OL, and Griffith M. (2017) DGIdb 3.0: a redesign and expansion of the drug-gene interaction database. Nucleic Acids Res, in press.

Feelders RA, Yasothan U, and Kirkpatrick P (2012) Pasireotide. Nat Rev Drug Discov 11:597-598.

Gong R (2014) Leveraging melanocortin pathways to treat glomerular diseases. Adv Chronic Kidney Dis 21:134-151.

Hebert SC, Mount DB, and Gamba G (2004) Molecular physiology of cation-coupled Cl- cotransport: the SLC12 family. Pflugers Arch 447:580-593.

Huang XP, Karpiak J, Kroeze WK, Zhu H, Chen X, Moy SS, Saddoris KA, Nikolova VD, Farrell MS, Wang S, et al. (2015a) Allosteric ligands for the pharmacologically dark receptors GPR68 and GPR65. Nature 527:477-483.

Huang Y, Skwarek-Maruszewska A, Horré K, Vandewyer E, Wolfs L, Snellinx A Saito T, Radaelli E, Corthout N, Colombelli J, et al. (2015b) Loss of GPR3 reduces the amyloid plaque burden and improves memory in Alzheimer's disease mouse models. Sci Transl Med 7:309ra164.

Hutchings CJ, Koglin M, Olson WC, and Marshall FH (2017) Opportunities for therapeutic antibodies directed at G-protein-coupled receptors. Nat Rev Drug Discov 16:787-810.

Insel PA, Wilderman A, Zambon AC, Snead AN, Murray F, Aroonsakool N, McDonald DS, Zhou S, McCann T, Zhang L, et al. (2015) G protein-coupled receptor (GPCR) expression in native cells: "Novel" endoGPCRs as physiologic regulators and therapeutic targets. Mol Pharmacol 88:181-187.

Jahny E, Yang H, Liu B, Jahnke B, Lademann F, Knösel T, Rümmele P, Grützmann R, Aust DE, Pilarsky C, et al. (2017) The G protein-coupled receptor RAI3 is an independent prognostic factor for pancreatic cancer survival and regulates proliferation via STAT3 phosphorylation. PLoS One 12:e170390.

Patel HH and Ostrom RS (2008) An orphan GPCR finds a home in the heart. Am J Physiol Heart Circ Physiol 295:H479-H481.

Pera T, Deshpande DA, Ippolito M, Wang B, Gavrila A, Michael JV, Nayak AP, Tompkins E, Farrell E, Kroeze WK, et al (2017) Biased signaling of the proton-sensing receptor OGR1 by benzodiazepines. FASEB J DOI: 10.1096/fj.201700555R [published ahead of print].

Rask-Andersen M, Masuram S, and Schiöth HB (2014) The druggable genome: evaluation of drug targets in clinical trials suggests major shifts in molecular class and indication. Annu Rev Pharmacol Toxicol 54:9-26.

Rebhan M, Chalifa-Caspi V, Prilusky J, and Lancet D (1998) GeneCards: a novel functional genomics compendium with automated data mining and query reformulation support. Bioinformatics 14:656-664.

Rimkus TK, Carpenter RL, Qasem S, Chan M, and Lo HW (2016) Targeting the sonic hedgehog signaling pathway: review of smoothened and GLI inhibitors. Cancers (Basel) 8:22.

Santos R, Ursu O, Gaulton A, Bento AP, Donadi RS, Bologa CG, Karlsson A, AlLazikani B, Hersey A, Oprea TI, et al. (2017) A comprehensive map of molecular drug targets. Nat Rev Drug Discov 16:19-34.

Southan C, Sharman JL, Benson HE, Faccenda E, Pawson AJ, Alexander SPH, Buneman OP, Davenport AP, McGrath JC, Peters JA, et al.; NC-IUPHAR (2016) The IUPHAR/BPS Guide to Pharmacology in 2016: towards curated quantitative interactions between 1300 protein targets and 6000 ligands. Nucleic Acids Res 44:D1054-D1068.

The UniProt Consortium (2017) UniProt: the universal protein knowledgebase Nucleic Acids Res 45:D158-D169.

Wacker D, Stevens RC, and Roth BL (2017) How ligands illuminate GPCR molecular pharmacology. Cell 170:414-427.

Wiley SZ, Sriram K, Liang W, Chang SE, French R, McCann T, Sicklick J, Nishihara H, Lowy AM, and Insel PA (2017) GPR68, a proton-sensing GPCR, mediates interaction of cancer associated fibroblasts and cancer cells. FASEB $J$ [published ahead of print].

Wishart DS, Knox C, Guo AC, Cheng D, Shrivastava S, Tzur D, Gautam B, and Hassanali M (2007) DrugBank: a knowledgebase for drugs, drug actions and drug targets. Nucleic Acids Res 36:D901-D906.

$\mathrm{Xie} \mathrm{H}$ and $\mathrm{He} \mathrm{SH}$ (2005) Roles of histamine and its receptors in allergic and inflammatory bowel diseases. World J Gastroenterol 11:2851-2857.

Yang Y, Fu A, Wu X, and Reagan JD (2012) GPR35 is a target of the loop diuretic drugs bumetanide and furosemide. Pharmacology 89:13-17.

Ye X, Tao Q, Wang Y, Cheng Y, and Lotan R (2009) Mechanisms underlying the induction of the putative human tumor suppressor GPRC5A by retinoic acid. Cancer Biol Ther 8:951-962.

Address correspondence to: Paul A. Insel, Department of Pharmacology, University of California San Diego, Mail Code 0636, La Jolla, CA 92093. E-mail: pinsel@ucsd.edu 